\title{
Mineralocorticoid modulation of central angiotensin-induced neuronal activity, water intake and sodium appetite
}

S.N. Thornton,

S.T. Omouessi and C. Falconetti
U684 UHP-INSERM, Faculté de Médecine, Université Henri Poincaré, Nancy, France

\section{Correspondence \\ S.N. Thornton \\ U684 UHP-INSERM \\ Faculté de Médecine \\ 9, Avenue de la forêt de Haye \\ B.P. 184 \\ 54505 Vandouvre Les Nancy cedex \\ France \\ Fax: $+33-3-8368-3639$ \\ E-mail: \\ simon.thornton@nancy.inserm.fr}

Presented at the International Symposium of Neuroendocrinology "Neuroendocrine control of body fluid homeostasis: past, present and future". Ribeirão Preto, SP, Brazil, September 1-3, 2006.

Received October 11, 2006 Accepted February 13, 2007

\begin{abstract}
Central angiotensin II (AngII) stimulates water and salt solution intake. Pretreatment with low-dose mineralocorticoid (DOCA) enhances this AngII-induced intake of salt solutions (the synergy theory) in Wistar and Sprague Dawley rats but not in Fischer rats. This response is mediated via the AT-1 receptor. Electrophysiological experiments using iontophoretic application of AngII and the AT-1 receptor-specific non-peptide antagonist losartan showed excitation of neurons in the preoptic/medial septum region of urethane-anesthetized male Wistar rats. DOCA pretreatment further enhances this neuronal excitation in response to AngII and reduces the responses to losartan. This generated the hypothesis that DOCA-enhanced AngIIinduced neuronal excitation is the neural support for the synergy theory. AT-2 receptors modulate these intake responses depending on sodium in the diet, and diuretic-induced dehydration during pregnancy produces a higher salt intake in the offspring. AngII-induced salt and water intakes were tested in offspring from Sprague Dawley mothers with only $1.8 \% \mathrm{NaCl}$ to drink in which half were treated with furosemide. The important observations were a) the AT- 1 antagonist alone suppressed intakes in offspring from mothers not treated with furosemide, b) both AT-1 and AT-2 antagonists suppressed intakes in offspring from furosemide-treated mothers, and c) combined administration of AT-1 and AT-2 antagonists greatly suppressed water intake in offspring from mothers not treated with furosemide. These results suggest that AT-1 and AT-2 receptors have variable properties (receptor number and/or second messengers). Furthermore, the activity and function of these central AngII receptors depend on the background mineralocorticoid levels. The exact mechanism of this influence, however, remains to be determined.
\end{abstract}

Behavior is a very good example of a motivated state and drinking behavior is perhaps one of the best examples. Not only is this particular behavior motivated by internal physiological signals but it can be initiated using components of these physiologi-
Key words

- Aldosterone

- Rat

- AT-1

- AT-2

- Iontophoresis cal signals. Drinking is just one aspect of body fluid regulation, an integrated physiological regulatory system that implicates nearly all the main organ networks of the body for a vital function, i.e., survival. It has even been postulated that without this inte- 
grated approach which includes the brain and a memory function, perhaps related to the physiological response to a disturbance in the body hydration state, the initial development of terrestrial life may not have occurred (1).

Ever since the first unicellular organisms formed on earth regulation of cellular water content, i.e., cellular volume, has been a never-ending problem. Water molecules move down along a concentration gradient until an equilibrium is reached or movement is prevented. Organisms living in an aqueous environment have to fight against either too much liquid coming in or loss of liquid to the outside depending on the osmolality of the surrounding fluids. Organisms, both plant and animal, living in fresh or sea water have developed mechanisms to combat these imposed movements of water across the various membranes in contact with the outside environment.

Land animals, and plants for that matter, have another problem, i.e., getting enough water in and keeping it there. As far as animals are concerned, water comes into the body from drinking and from the metabolism of ingested food, and then the renal system, and to some extent the respiratory system, are responsible for keeping it there. Once the water is in, the internal distribution must be assured by yet another system, i.e., the cardiovascular one, with all its inherent control mechanisms. However, many other regulatory physiological systems depend also on the water ingested and all need separate control mechanisms. The interplay between all of these mechanisms gives a fascinating picture of physiological control.

In his early work on rats James Fitzsimons (2) described two major thirst states, primary and secondary, the former being a state of physiological need and thus regulatory in nature, and the latter being not principally regulatory in nature.

Primary thirst was subsequently further divided into thirst of intracellular origin and thirst of extracellular origin. Under ideal conditions where all physiological systems function correctly, the osmolality of all fluid compartments is the same and water is distributed proportionally (two thirds intracellular and one third extracellular). Another element that plays a key role in the regulation of body fluids is the sodium ion. Sodium is the principal cation of the extracellular fluids (potassium being that of the intracellular compartment) and thus plays an essential role in the regulation of body fluid osmolality, i.e., the gradient that regulates the movement of water.

As far as extracellular thirst is concerned, in an ideal state, fluid would be lost initially from the vasculature leading to a decrease in blood volume. As the volume loss increases fluid would also move out of the interstitial compartment into the vasculature. The overall regulation of blood volume and blood pressure is complex. In the case of thirst and fluid regulation it could be simplified initially to the control of blood volume. There are detectors in the great veins for a decrease in volume that signal to the brain to search for water and to increase the release of antidiuretic hormone. These receptors also cause an increase in sodium appetite and reflex release of renin through sympathetic activation. Furthermore, in the kidney other volume/pressure detectors sense the decrease in perfusion pressure of the kidney and cause further release of renin. This enzyme acts on circulating angiotensinogen, an $\alpha_{2}$-globulin released from the liver, forming the decapeptide angiotensin I. Angiotensin-converting enzyme in the lung converts this to the octapeptide angiotensin II (AngII) that has several actions. It is vasoconstrictive (via a direct and indirect action on the vascular wall) thus reducing the diameter of the vasculature to fit the lower blood volume and to facilitate blood flow, it stimulates the thirst centers in the brain to search out and ingest water, and it stimulates the release of the mineralocorticoid hormone aldosterone from 
the adrenals.

Aldosterone is the hormone of sodium regulation and has two principal actions, one to increase sodium absorption in the kidney via an action on the distal tubule and the other to sensitize certain specific areas of the brain (especially the hypothalamus) to the circulating levels of AngII, the combined effect of which is to stimulate sodium appetite $(2,3)$.

The ingestion of water and sodium together restores the lost blood volume to the initial values (2). As blood volume increases, the signals to stimulate thirst, antidiuretic hormone release and the release of renin are thus decreased. The action of aldosterone, on the other hand, appears to continue for some time, even after its levels in the blood have decreased to normal values, as is the case for most steroid hormones, since they activate many intracellular pathways including protein synthesis.

The homeostatic regulation of hydromineral balance is thus designed to cope perfectly well with variations in water intake through regulation of blood vessel caliber, regulation of water and sodium excretion through the kidneys and regulation of the behavioral act, i.e., thirst and sodium appetite.

One of the key components of this physiologically regulated system is AngII which, when administered into the brain either directly into various brain structures or into the cerebro-ventricular system, stimulates the intake of water and of concentrated $\mathrm{NaCl}$ solution. This action is mediated via angiotensin type 1 receptors (AT-1) $(3,4)$ present throughout the hypothalamus and specifically in the circumventricular organs around the 3rd cerebral ventricle, structures implicated in the brain sensing of the body's hydrational state.

Electrophysiological studies of neurons with these receptors have shown a multitude of responses. In patch clamp studies with bath application AngII increases the sponta- neous activity of these neurons (5). Iontophoretic application directly onto neurons has shown both an increase and a decrease in activity (6-8), as well as an increase in field potentials (9).

Treatment with aldosterone had been shown to increase the number of binding sites for AngII centrally (10-12) and a short treatment ( 3 to 4 days) with a low dose of the synthetic mineralocorticoid (DOCA) enhances AngII-induced drinking of hypertonic salt solutions (13). These results came to form the basis of the synergy theory developed by Epstein and his colleagues (14), whereby it was the uniquely central interaction of these two hormones that led to the enhancement of the appetite for sodium. This was found to be the case in Wistar and Sprague Dawley rats but not in Fischer (15) or Zucker obese rats (Omouessi ST, personal communication), suggesting that perhaps the mechanism was not applicable to all rats. The use of receptor-specific antagonists showed that this response was mediated via the AT-1 receptor (16).

Electrophysiological experiments using iontophoretic application of AngII and the AT-1 receptor-specific non-peptide antagonist losartan, showed that both substances increased excitation of neurons in the preoptic/medial septum region of urethane-anesthetized male Wistar rats (8). The increase in activity in response to losartan was interpreted as the inhibition of an AngII-induced inhibition (7), yielding excitation (8). DOCA pretreatment further enhanced this neuronal excitation in response to AngII but reduced the responses to losartan (17). This generated the hypothesis that DOCA-enhanced AngII-induced neuronal excitation was the neural support for the synergy theory's-enhanced salt intake. Furthermore, it has been taken as evidence that there is possibly more than just one type, or functional state, of the AT-1 receptor (17) since the response to locally applied losartan went from frequent short- and long-term neuronal excitation be- 
fore DOCA to infrequent short-term excitation only, and in rare cases inhibition, afterwards (8). As if the mineralocorticoid treatment had modified the type of AngII receptor responding, or its functional state $(18,19)$.

Liénard et al. (20) took this one step further by using behavioral experiments with losartan and DOCA. They argued that iontophoretic application would have put very small quantities of losartan around the neurons, similar to what occurs with AngII, and that therefore very low doses of this particular antagonist should be used. The results showed that in animals already tested with central injections of $10 \mathrm{pmol}$ AngII, which generated a significant intake of water and a small intake of $2 \%$ salt solution, losartan at the same Mol for Mol dose generated an appetite for sodium over the 2 days following administration. Once again suggesting that inhibition of a local inhibition could generate an intake. Furthermore, treatment of these rats with DOCA given peripherally after the central administration of losartan produced an appetite for the concentrated salt solution, something which did not happen if DOCA was given before losartan. This was interpreted to indicate that the normal action of the mineralocorticoid was to modify the central functioning of AngII receptors such that those responsible for the inhibition were no longer functioning. This would be in agreement with the findings of a reduced action of losartan in DOCA-treated rats (17), again suggesting that there is a tonic inhibitory signal against a salt appetite but when this is removed, either with DOCA pretreatment or with central losartan, then AngII can stimulate an increased intake of sodium.

This was tested further in Fischer rats known to have a reduced appetite for sodium and who respond minimally to challenges that would normally increase sodium appetite. Using the electrophysiological approach with iontophoretic application we observed that neurons in the forebrain of these rats were much less responsive to locally applied AngII than in Wistar rats (21). Furthermore, local application of losartan produced inhibition only and never excitation, as has been observed in Wistar rats $(8,17)$. These results suggested once again that it could be the state of activity, or excitability to local AngII that is the neuronal basis of sodium appetite. The Fischer rats with a very much reduced excitability had a reduced appetite. However, in the same experiments reported by Falconetti et al. (21), the authors observed that central injection of AngII into conscious behaving animals produced equivalent intakes of concentrated sodium solution in Fischer and Wistar rats. This would suggest that motivated behavior is not so simple to analyze and that, although the forebrain may be the center of body fluid intake regulation, the neuronal substrate of this regulation is slightly more complex than analysis of the activity of just one area (relatively large as it might appear; see Ref. 22, page 171).

In a subsequent study on Fischer rats, again looking at behavior of both the intact, non-anesthetized animal and neurons in the forebrain of anesthetized animals, it was observed that DOCA pretreatment could increase the responsiveness of forebrain neurons to AngII, as reported previously in Wistar rats, but that the salt drinking responses to centrally applied AngII were not enhanced (23). Thus, the two phenomena can be separated and perhaps enhancement can be observed depending on the degree of increased excitability, or degree of decreased inhibition, of the forebrain neurons. Experiments using increasing doses of DOCA pretreatment of Fischer rat remain to be carried out.

Two major subtypes of the AngII receptors have been identified, AngII type-1 (AT1) and AngII type-2 (AT-2), with the development of specific non-peptide antagonists (24-27). These receptors have been shown to have different distributions and functions within the central nervous system (28-30). 
Although, as mentioned previously, all major functions attributed to central administration of AngII have been shown to be mediated through the AT-1 receptor, the possible role of the AT-2 receptor as a mediator of AT-1 function has been suggested by several experiments. In adrenalectomized rats, having no plasma aldosterone, central losartan injection decreased sodium intake in a dose-response manner (31). The AT-2 specific antagonist PD 123319 was not effective by itself, but when combined with losartan, both at a low dose, there was synergy of action and a more pronounced antagonism of the appetite than simple addition of the effect of the individual doses (31). This was one of the first suggestions that the two receptor subtypes could have an interaction in the central nervous system. Subsequent research has even gone so far as to indicate that the AT- 2 receptor could be an antagonist of the AT-1 receptor (32).

The distribution and function of AngII receptors in the brain has been shown to be influenced by plasma aldosterone levels ( 10 , $11,17)$, as well as by the sodium content of the diet $(33,34)$. The latter finding suggests that sodium in the food would have an influence on plasma aldosterone levels which could thus influence central AngII receptor function.

Furthermore, an extracellular dehydration event during pregnancy in both rats and humans predisposes the offspring to a higher preference for sodium in later life $(35,36)$. This effect is dependent on the dehydrationinduced maternal aldosterone crossing the placental barrier and altering the sensitivity of the fetal brain angiotensin receptors (37). Although it has been hypothesized that the sensitivity of brain AngII receptors of these offspring is altered by the dehydration event in the mother, few studies have been undertaken to investigate animals thus produced.

In recent as yet unpublished study, it has been shown that in male offspring from pregnant Sprague Dawley rats given only $1.8 \%$
$\mathrm{NaCl}$ solution to drink (in order to reduce blood aldosterone levels), with half of the pregnant dams receiving extracellular dehydration by injection of furosemide twice towards the end of pregnancy, AngII-induced intakes of water and a concentrated salt solution were modulated by prior injection of AT-1 and/or AT-2 receptor-specific antagonists; a) AT-1 but not AT-2 receptor blockade alone suppressed water and $1.8 \% \mathrm{NaCl}$ intake in male offspring from mothers not treated with furosemide; b) both AT-1 and AT-2 receptor antagonists suppressed water and $1.8 \% \mathrm{NaCl}$ intake in offspring from furosemide-treated mothers, and c) combined administration of AT-1 and AT-2 receptor antagonists greatly suppressed water (and to a lesser extent salt) intake only in offspring from mothers not treated with furosemide (Falconetti C, Omouessi ST and Thornton $\mathrm{SN}$, unpublished results).

It would thus appear that the absence of mineralocorticoids during pregnancy permitted an interaction of both AT-1 and AT2 receptors, perhaps in the form of a heterodimer, suggesting that, under these particular conditions, AT-2 could well be a modulator of AT-1 activity. Furthermore, the transient increase in mineralocorticoids during pregnancy appeared to be sufficient to modulate the function of the two receptors so that they now were observed to play an equal role in the response to centrally administered AngII. Again it could be suggested that a dimer structure is possible but this time antagonism of one of the pair inhibits also the other.

At least two studies have indicated that the $\mathrm{G}$ protein activated or the second messengers used can vary depending on the AT1 receptor activated $(38,39)$ or more precisely the type of neuron stimulated, so that the possibilities of modulation become very complex. It would thus appear that mineralocorticoids are able to modulate not only receptor expression but also receptor function. Suffice it to say that the interaction of 
the brain angiotensin receptor system with the mineralocorticoids is complex and will lead to some interesting discoveries in the future.

In conclusion, the regulation of body fluids is an integrated organization of various systems to bring water and sodium in, to keep the substances there and to distribute them throughout all fluid compartments of the body. These systems are known and have been studied intensely over the past 30 years or so. The hormones implicated in this vital regulatory function are also known but their complexity of interaction is beginning to shed light on as yet unstudied physiologi- cal mechanisms that are also part of this regulation. This review has dealt with what happens in the central nervous system but there is also an interaction of mineralocorticoids with the peripheral renin angiotensin system that may be just as complex, if not more so, and probably responsible for the cardiovascular dysfunction seen in many hypertensive disease states. The presence therein of elevated levels of these two particular hormones, AngII and aldosterone, may very well be the consequence of the normal physiological response to long-term mild hypovolemia.

\section{References}

1. Denton DA, McKinley MJ, Weisinger RS. Hypothalamic integration of body fluid regulation. Proc Natl Acad Sci U S A 1996; 93: 73977404.

2. Fitzsimons JT. Angiotensin, thirst, and sodium appetite. Physiol Rev 1998; 78: 583-686.

3. Johnson AK. Brain mechanisms in the control of body fluid homeostasis. In: Gisolfi CV, Lamb DR (Editors), Perspectives in exercise science and sports medicine, fluid homeostasis during exercise. Indianapolis: Benchmark Press; 1990. p 347-419.

4. Beresford MJ, Fitzsimons JT. Intracerebroventricular angiotensin IIinduced thirst and sodium appetite in rat are blocked by the AT1 receptor antagonist, Losartan (DuP 753), but not by the AT2 antagonist, CGP 42112B. Exp Physiol 1992; 77: 761-764.

5. Li Z, Ferguson AV. Angiotensin II responsiveness of rat paraventricular and subfornical organ neurons in vitro. Neuroscience 1993; 55: 197-207.

6. Huwyler T, Felix D. Angiotensin II-sensitive neurons in septal areas of the rat. Brain Res 1980; 195: 187-195.

7. Simonnet G, Bioulac B, Rodriguez F, Vincent JD. Evidence of a direct action of angiotensin II on neurones in the septum and in the medial preoptic area. Pharmacol Biochem Behav 1980; 13: 359363.

8. Thornton SN, Nicolaidis S. Long-term mineralocorticoid-induced changes in rat neuron properties plus interaction of aldosterone and ANG II. Am J Physiol 1994; 266: R564-R571.

9. von Bohlen Und Halbach O, Albrecht D. The CNS renin-angiotensin system. Cell Tissue Res 2006; 326: 599-616.

10. Gutkind JS, Kurihara M, Saavedra JM. Increased angiotensin II receptors in brain nuclei of DOCA-salt hypertensive rats. $A m \mathrm{~J}$ Physiol 1988; 255: H646-H650.

11. King SJ, Harding JW, Moe KE. Elevated salt appetite and brain binding of angiotensin II in mineralocorticoid-treated rats. Brain Res 1988; 448: 140-149.
12. Shelat SG, King JL, Flanagan-Cato LM, Fluharty SJ. Mineralocorticoids and glucocorticoids cooperatively increase salt intake and angiotensin II receptor binding in rat brain. Neuroendocrinology 1999; 69: 339-351.

13. Fluharty SJ, Epstein AN. Sodium appetite elicited by intracerebroventricular infusion of angiotensin II in the rat: II. Synergistic interaction with systemic mineralocorticoids. Behav Neurosci 1983; 97: 746-758.

14. Sakai RR, Nicolaidis S, Epstein AN. Salt appetite is suppressed by interference with angiotensin II and aldosterone. Am J Physiol 1986; 251: R762-R768.

15. Rowland NE, Fregly MJ. Behavioral and physiological aspects of body fluid homeostasis in Fischer 344 rats. Physiol Behav 1988; 42: 499-505.

16. Cooney AS, Fitzsimons JT. The effect of the putative AT2 agonist, paminophenylalanine6 angiotensin II, on thirst and sodium appetite in rats. Exp Physiol 1993; 78: 767-774.

17. Martial FP, Thornton SN, Lienard F, Mousseau MC, Nicolaidis S. Tonic neuronal inhibition by All revealed by iontophoretic application of Losartan, a specific antagonist of All type-1 receptors. Brain Res Bull 1994; 34: 533-539.

18. Wilson KM, Sumners C, Hathaway S, Fregly MJ. Mineralocorticoids modulate central angiotensin II receptors in rats. Brain Res 1986; 382: 87-96.

19. Noda K, Feng YH, Liu XP, Saad Y, Husain A, Karnik SS. The active state of the AT1 angiotensin receptor is generated by angiotensin II induction. Biochemistry 1996; 35: 16435-16442.

20. Lienard F, Thornton SN, Martial FP, Mousseau MC, Nicolaidis S. Angiotensin II receptor subtype antagonists can both stimulate and inhibit salt appetite in rats. Regul Pept 1996; 66: 87-94.

21. Falconetti C, Chapleur M, Fernette B, Thornton SN. Central All evokes a normal sodium appetite in the Fischer rat, but its low spontaneous sodium intake may be related to reduced excitation 
and increased inhibition in septo-preoptic All neurons. Brain Res Bull 2004; 62: 405-412.

22. Mousseau MC, Thornton SN, Lienard F, Martial FP, Nicolaidis S. Water versus salty taste and iontophoretic ANGII responses of septopreoptic neurons in dehydrated and euhydrated awake rats. Brain Res Bull 1996; 41: 167-173.

23. Omouessi ST, Falconetti C, Chapleur M, Fernette B, Thornton SN. Mineralocorticoid pretreatment enhances angiotensin II-induced neuronal excitation but not salt drinking in male Fischer rats. $J$ Neuroendocrinol 2007; 19: 109-115.

24. Chiu AT, Herblin WF, McCall DE, Ardecky RJ, Carini DJ, Duncia JV, et al. Identification of angiotensin II receptor subtypes. Biochem Biophys Res Commun 1989; 165: 196-203.

25. Dudley DT, Panek RL, Major TC, Lu GH, Bruns RF, Klinkefus BA, et al. Subclasses of angiotensin II binding sites and their functional significance. Mol Pharmacol 1990; 38: 370-377.

26. Kakar SS, Sellers JC, Devor DC, Musgrove LC, Neill JD. Angiotensin II type-1 receptor subtype cDNAs: differential tissue expression and hormonal regulation. Biochem Biophys Res Commun 1992; 183: 1090-1096.

27. Timmermans PB, Wong PC, Chiu AT, Herblin WF, Benfield P, Carini DJ, et al. Angiotensin II receptors and angiotensin II receptor antagonists. Pharmacol Rev 1993; 45: 205-251.

28. Chang RS, Lotti VJ, Chen TB, Faust KA. Two angiotensin II binding sites in rat brain revealed using [125I]Sar1, Ile8-angiotensin II and selective nonpeptide antagonists. Biochem Biophys Res Commun 1990; 171: 813-817.

29. Song K, Allen AM, Paxinos G, Mendelsohn FA. Mapping of angiotensin II receptor subtype heterogeneity in rat brain. J Comp Neurol 1992; 316: 467-484.

30. Toney GM, Porter JP. Functional roles of brain AT1 and AT2 receptors in the central angiotensin II pressor response in conscious young spontaneously hypertensive rats. Brain Res Dev Brain Res 1993; 71: 193-199.

31. Galaverna O, Polidori C, Sakai RR, Lienard F, Chow SY, Fluharty SJ. Blockade of central angiotensin II type 1 and type 2 receptors suppresses adrenalectomy-induced $\mathrm{NaCl}$ intake in rats. Regul Pept 1996; 66: 47-50.

32. AbdAlla S, Lother $\mathrm{H}$, Abdel-tawab AM, Quitterer U. The angiotensin II AT2 receptor is an AT1 receptor antagonist. J Biol Chem 2001; 276: 39721-39726.

33. Sandberg K, Ji H, Catt KJ. Regulation of angiotensin II receptors in rat brain during dietary sodium changes. Hypertension 1994; 23 (Suppl): I-137-I-141.

34. Camara AK, Osborn J. Central AT1 and AT2 receptors mediate chronic intracerebroventricular angiotensin II-induced drinking in rats fed high sodium chloride diet from weaning. Acta Physiol Scand 2001; 171: 195-201.

35. Nicolaidis S, Galaverna O, Metzler $\mathrm{CH}$. Extracellular dehydration during pregnancy increases salt appetite of offspring. Am J Physiol 1990; 258: R281-R283.

36. Crystal SR, Bernstein IL. Morning sickness: impact on offspring salt preference. Appetite 1995; 25: 231-240.

37. Galaverna O, Nicolaidis S, Yao SZ, Sakai RR, Epstein AN. Endocrine consequences of prenatal sodium depletion prepare rats for high need-free $\mathrm{NaCl}$ intake in adulthood. Am J Physiol 1995; 269: R578-R583.

38. Crawford KW, Frey EA, Cote TE. Angiotensin II receptor recognized by DuP753 regulates two distinct guanine nucleotide-binding protein signaling pathways. Mol Pharmacol 1992; 41: 154-162.

39. Daniels D, Yee DK, Faulconbridge LF, Fluharty SJ. Divergent behavioral roles of angiotensin receptor intracellular signaling cascades. Endocrinology 2005; 146: 5552-5560. 